\title{
Efficacy Comparison and Safety Analysis of Radical Resection of Sigmoid Colon Cancer Based on Different Cancer Locations
}

\section{Li Chen}

Zhejiang University School of Medicine Sir Run Run Shaw Hospital

\section{Engeng Chen}

Zhejiang University School of Medicine Sir Run Run Shaw Hospital

Dongai Jin

Zhejiang University School of Medicine Sir Run Run Shaw Hospital

\section{Min Chen}

Zhejiang University School of Medicine Sir Run Run Shaw Hospital

\section{Wei Zhou}

Zhejiang University School of Medicine Sir Run Run Shaw Hospital zhangfa song ( $\nabla$ songzhangfa@zju.edu.cn )

Zhejiang University https://orcid.org/0000-0001-6301-1813

\section{Research Article}

Keywords: sigmoid colon cancer, surgery, safety, anastomosis

Posted Date: September 28th, 2021

DOI: https://doi.org/10.21203/rs.3.rs-489873/v3

License: (1) This work is licensed under a Creative Commons Attribution 4.0 International License. Read Full License 


\section{Efficacy comparison and safety analysis of radical resection of sigmoid colon cancer based on different cancer locations}

Li Chen ${ }^{1}$, Engeng Chen ${ }^{1}$, Dongai Jin ${ }^{1}$, Min Chen ${ }^{1}$, Wei Zhou ${ }^{1}$, Zhangfa Song ${ }^{1}$

${ }^{1}$ Li Chen, M.D. 3415021@zju.edu.cn;

${ }^{1}$ Engeng Chen, M.D. chenengeng@zju.edu.cn; ${ }^{1}$ Dongai Jin, M.D.3203068@zju.edu.cn;

${ }^{1}$ Min Chen, M.D. chenmin@zju.edu.cn; ${ }^{1}$ Wei Zhou, Ph.D. zhouwei@zju.edu.cn;

${ }^{1}$ Zhangfa Song, Ph.D. songzhangfa@zju.edu.cn

${ }^{1}$ Department of Colorectal Surgery, Sir Run Run Shaw Hospital, School of Medicine, Zhejiang University, Hangzhou, China.

Address Correspondence to: Zhangfa Song, Department of Colorectal Surgery, Sir Run Run Shaw Hospital, School of Medicine, Zhejiang University, Hangzhou, China. E-mail: songzhangfa@zju.edu.cn Tel: 13616533922 


\begin{abstract}
:
Background: Sigmoid colon cancer is a common type of colon cancer; it refers to tumor lesions occurring in the segment approximately $16 \mathrm{~cm}$ to $50 \mathrm{~cm}$ from the anal margin. Currently, surgical resection is the most effective treatment for non-metastatic sigmoid colon cancer. Therefore, to more accurately standardize colon cancer surgery, we classified the sigmoid colon into distal and proximal segments. This study compares and analyzes the intraoperative situation, postoperative pathology and safety of radical resection of sigmoid cancer in different locations from different aspects. Result: The patients with sigmoid colon tumor can be divided into distal group (16-30 cm from the anus) and proximal group (31-50 cm from the anus) according to the distance between the tumor and the anal margin. Conclusion: The patients in the distal group using stapler for intestinal anastomosis and anal decompression tube were significantly more than those in the proximal group. For the ligation of the inferior mesenteric artery, high ligation was usually used or the left colic artery was usually preserved in the distal group, and the superior rectal artery was usually retained in the proximal group. The incidence of postoperative complications in the distal group was higher than that in the proximal group, but there was no significant difference between the two groups.
\end{abstract}

Keywords: sigmoid colon cancer, surgery, safety, anastomosis

What does this paper add to the literature: Sigmoid colon covers a wide range, from $15 \mathrm{~cm}$ to $50 \mathrm{~cm}$ from the anus. Therefore, in order to standardize the operation of sigmoid colon cancer more accurately, we classified the sigmoid colon into distal and proximal segments and compared the efficacy and safety from two parts. 


\section{Introduction}

Colon cancer is one of the most common digestive tract cancers. In recent years, incidence rate and mortality rate has been increasing worldwide, and it has become an important cause threatening human health [1]. In 2018, there were 1.8 million new colorectal cancer cases and 880000 deaths worldwide [2]. According to relevant studies, the number of colon cancer deaths in the world will reach 1.1 million every year by 2030 [1-3]. Colorectal cancer is the fifth most common malignancy in China. With the change of diet habits and lifestyle, the incidence rate of colon cancer has exceeded that of rectal cancer.

Laparoscopic surgery for colon cancer was introduced in the late 1990s. In the past two decades, robotic surgery has also been widely used in the treatment of colon cancer [4-6]. With the increase in minimally invasive surgical approaches and the advancement of surgical instruments, colorectal surgery has become increasingly concerned with the disease itself.

The sigmoid colon connects the descending colon at the left iliac crest, turns into the pelvic cavity along the left iliac fossa, and is curved in an "s" shape for its entire length. The sigmoid colon continues to the rectum at the level of the third sacral vertebra and has a length of approximately 30 $\mathrm{cm}$ to $40 \mathrm{~cm}$. Sigmoid colon cancer is a common type of colon cancer; it refers to tumor lesions occurring in the segment approximately $16 \mathrm{~cm}$ to $50 \mathrm{~cm}$ from the anal margin. Currently, surgical resection is the most effective treatment for non-metastatic sigmoid colon cancer [3].

Due to the many subtypes of the inferior mesenteric artery and the influence of the position of the sigmoid colon cancer, anastomosis, vascular ligation, and lymph node dissection during radical resection may vary, and analyses and summaries of these differences are important for optimizing colorectal surgery [7,8]. This study compares and analyzes the intraoperative situation, postoperative pathology and safety of radical resection of sigmoid cancer in different locations from different aspects.

\section{Methods}

\section{Study subjects and general information}

Patients who underwent laparoscopic radical resection of sigmoid colon cancer at Sir Run Run Shaw Hospital affiliated with the School of Medicine, Zhejiang University, between January 2019 and March 2020 were enrolled in the study. The general information of the patients comprised all of the written case materials provided by the Medical Records Room of Sir Run Run Shaw Hospital. This study was approved by the ethics committee of our hospital. We confirm that all methods were performed in accordance with the relevant guidelines and regulations.

The inclusion criteria for this study were patients with clear histopathology of sigmoid adenocarcinoma. Colonic lesions were measured by electronic colonoscopy and were located $16 \mathrm{~cm}$ to $50 \mathrm{~cm}$ from the anus. Patients were diagnosed with nondistant metastasis or distant metastasis according to the preoperative evaluation and underwent surgery for the primary lesion, and none of the patients received neoadjuvant therapy before surgery.

The exclusion criteria for this study were patients who underwent surgery for the recurrence of colorectal cancer; patients with multiple tumors; patients who received preoperative radiotherapy 
and chemotherapy; and patients with postoperative pathology suggestive of melanoma, neuroendocrine carcinoma and other non-adenocarcinoma.

According to the inclusion and exclusion criteria, a total of 35 patients were included in this study. The parameters of 35 patients were collected based on medical records and included age, sex, preoperative body mass index (BMI), carcinoembryonic antigen (CEA) level before surgery, and tumor location. BMI is a common measure of obesity and is calculated as weight $(\mathrm{kg}) \div$ height squared $\left(\mathrm{m}^{2}\right)$. Tumor position refers to the distance between the anal margin and the lower edge of the tumor as measured by electronic colonoscopy.

\section{Surgery}

All patients underwent radical surgery. Laparoscopic radical resection for sigmoid colon cancer was performed by a qualified colorectal surgery specialist. None of the patients included in this study received permanent or prophylactic stoma.

\section{Pathological analysis}

After the specimens were macroscopically examined by the operating room physician, each specimen was sent for pathological examination. All specimens of patients with sigmoid colon cancer were examined by colorectal cancer pathologists in our hospital. The pathological stage of the tumor was determined according to the eighth edition of the American Joint Committee on Cancer (AJCC). The degree of tumor differentiation, the total number of detected lymph nodes and positive lymph nodes, and the number of lymph nodes dissected at the root of the inferior mesenteric vessels were evaluated.

\section{Observational index}

The anastomosis method, ligation method, surgical duration and intraoperative blood loss were recorded in the two groups. The time of postoperative feeding and exhaust, length of hospital stay, and complications were compared between the two groups. Video recordings of the surgery were used to determinethe branches of the inferior mesenteric artery and the vascular management time. The anastomosis methods mainly included manual anastomosis and stapler anastomosis. The method of blood vessel ligation was divided into three categories: high ligation of the inferior mesenteric artery, left colic artery preservation and superior rectal artery preservation. Surgical duration refers to the time from the establishment of pneumoperitoneum to the end of abdominal closure. Intraoperative blood loss was calculated based on the total amount of blood collected in the suction bottle before the pelvic irrigation plus the total amount of blood in the gauze. The total amount of blood in the gauze was the weight of the wet gauze minus the weight of the dry gauze. For the convenience of calculation, the amount of blood in the gauze was calculated as $1 \mathrm{~g}$ equals $1 \mathrm{ml}$. The vascular management time was measured from the isolation of the inferior mesenteric artery until the inferior mesenteric vein was transected and lymph node dissection was completed.

\section{Statistical analysis}

Continuous variables are described as mean values and standard deviations, and categorical variables are described as numbers of patients and percentages. Correlation analyses between groups were conducted using the $t$ test and the chi-square test. The median distance of the sigmoid colon cancer from the anus for all study subjects was $20 \mathrm{~cm}$. Comparative analysis showed that it was appropriate to use $30 \mathrm{~cm}$ as the cutoff value for the proximal and distal margins. The patients were divided into two groups: the distal group $(16-30 \mathrm{~cm}$ from the anus) and the proximal group (31-50 $\mathrm{cm}$ from the anus). $\mathrm{P}<0.05$ was considered statistically significant. All data were analyzed using SPSS 23.0. 


\section{Results}

\section{Classification of the inferior mesenteric artery branches}

Based on the relationship between the left colic artery, the sigmoid artery, and the superior rectal artery, the Japanese scholars Munoro et al. classified the inferior mesenteric artery into types I-IV (Figure): in type I, the left colic artery arises independently from the inferior colonic artery, and the sigmoid artery and the superior rectal artery arise from a common trunk (Figure A); in type II, the inferior mesenteric artery first divides into one branch, which is the common trunk of the left colic artery and the sigmoid artery (Figure B); in type III, the left colic artery, sigmoid artery, and superior rectal artery branch at the same location (Figure C); and in type IV, the left colic artery is absent $[9,10]$. By reviewing the surgical video, we performed an analysis of the inferior mesenteric artery subtypes of 35 patients with sigmoid colon cancer. We found 18 cases $(51.4 \%)$ of type I inferior mesenteric artery, 10 cases (28.6\%) of type II, and 7 cases (20\%) of type III.

\section{Selection of the cutoff points for the location of sigmoid colon cancer}

The patients with cancer of the sigmoid colon $(16 \mathrm{~cm}-50 \mathrm{~cm}$ from the anus) were divided into two groups based on the cancer location of the sigmoid colon. Four different cutoff points were tested (Table 1). It was found that $30 \mathrm{~cm}$ was an appropriate the cutoff point between the distal group and the proximal group.

\section{Patient population and clinical information}

The patients were divided into two groups: the distal group (16-30 $\mathrm{cm}$ from the anus) and the proximal group (31-50 $\mathrm{cm}$ from the anus). There was no significant difference in age, sex, and preoperative BMI between the two groups (Table 2). The CEA level before surgery was significantly higher in the proximal group than in the distal group $(\mathrm{P}=0.019)$.

\section{Intraoperative situation of radical resection for sigmoid colon cancer}

The patients were divided into the distal group (16-30 $\mathrm{cm}$ from the anus) and the proximal group (31-50 cm from the anus). There was no significant difference between the two groups in the time required for the management of the inferior mesenteric vessels, the total operation time, or the intraoperative blood loss (Table 3). In the distal group, the number of patients who underwent stapler anastomosis and retained anal decompression tube was significantly higher than that in the proximal group $(\mathrm{P}=0.002)$. For the ligation of the inferior mesenteric artery, high ligation was usually used or the left colic artery was usually preserved in the distal group, and the superior rectal artery was usually retained in the proximal group, and the difference between the two groups was significant $(\mathrm{P}<0.001)$.

\section{Pathological analysis after radical resection of sigmoid colon cancer}

Pathological stage 0 refers to patients with a cancerous lesion after local resection under colonoscopy, but no pathological residual tumor was observed in the pathological analysis after radical resection. The patients were divided into the distal group (tumor $16-30 \mathrm{~cm}$ from the anus) and the proximal group (tumor $31-50 \mathrm{~cm}$ from the anus). There was no significant difference between the two groups in the postoperative tumor pathological stage, pathological tissue differentiation, total number of lymph node dissections, total number of positive lymph nodes, or 
number of dissections at the root of the inferior mesenteric vessels (Table 4). After formalin fixation of the pathological specimens from two groups, the length of the bowel was significantly shorter in the distal group than in the proximal group $(\mathrm{P}=0.006)$.

\section{Postoperative recovery and complications}

There was no statistical difference between the two groups in terms of postoperative recovery, time to exhaust, time to fluid intake, drainage tube removal time, and postoperative hospital stay (Table 5).The incidence of postoperative complications in the distal group (two cases of abdominal infection, one case of intestinal obstruction, one case of chylous fistula) was higher than that in the proximal group (one case of intestinal obstruction), but there was no significant difference between the two groups.

\section{Discussion}

Sigmoid colon cancer is a type of colon cancer located at the junction of the rectum and descending colon. The age of incidence is mainly between 60 and 70 years [1-3]. The early clinical manifestations of sigmoid colon cancer are mainly abdominal distension, abdominal pain, changes in stool characteristics and habits, hematochezia, etc. With the progression of the disease, gastrointestinal obstruction and other related symptoms may occur. Due to the lack of specificity of early symptoms, sigmoid colon cancer is easily confused with gastrointestinal-related diseases such as irritable bowel syndrome and mixed hemorrhoids. Finally, with the prolongation of the disease course, the difficulty of treatment greatly increases. Currently, surgical resection is the main treatment for sigmoid colon cancer in clinical practice. The traditional surgical approach is mainly open surgery, which has the problems of a long operation time, slow recovery, and great trauma to the patient. Currently, with the development of medical science and technology, minimally invasive surgical approaches such as laparoscopy and robotic surgery have been continuously applied in clinical practice. As a minimally invasive medical technique [4-6], laparoscopic surgery can make full use of camera technology to explore abdominal lesions and has advantages such as reduced bleeding and rapid postoperative recovery. In a laparoscopic abdominal operation, it is easier for the surgeon to find the space under Toldt's fascia, which can effectively prevent accidental access to other spaces and reduce damage to the blood vessels, ureter, and related nerves during surgery, thus effectively reducing bleeding and protecting patients' voiding function, sexual function, and bowl movement function [6].

Sigmoid colon cancer is different from rectal cancer or right-sided colon cancer and covers a wide range, from $15 \mathrm{~cm}$ to $50 \mathrm{~cm}$ from the anus. Therefore, to more accurately standardize colon cancer surgery, we classified the sigmoid colon into distal and proximal segments. At present, anastomosis methods for colon cancer mainly include stapler anastomosis and manual anastomosis in radical resection [11]. By conducting a statistical analysis of the intraoperative conditions, we found that due to the different anatomical structures of the two groups, the number of patients who underwent stapler anastomosis with a retained anal decompression tube was significantly higher in the distal group than in the proximal group $(\mathrm{P}=0.002)$. Similarly, due to the different tumor locations and the impact of vascular retraction, the methods of inferior mesenteric artery ligation 
also differed [12,13]. High ligation or left colic artery preservation was usually used in the distal group, while the superior rectal artery was usually retained in the proximal group; the difference between the two groups was significant $(\mathrm{P}<0.001)$. There was no statistically significant difference in the other operative times or intraoperative blood loss volumes.

With the proposed and extensive application of total mesorectal excision and complete mesenteric excision, the local recurrence rate and five-year survival rate of colorectal malignancies have significantly improved. The need to complete D3 lymph node dissection of tumors during radical resection of rectal and sigmoid colon cancers, based on the experience of Japan and other countries, is still controversial [12-16]. Currently, the boundary of lymph nodes at the root of the inferior mesenteric artery is generally defined as medial to the superior mesenteric artery, lateral to the inferior mesenteric vein, caudate to the left colic artery root, and cephalic to the inferior mesenteric artery root [17]. Reports in the past 20 years show that the positive rate of lymph nodes at the root of the inferior mesenteric artery in patients with rectal cancer and sigmoid colon cancer was $0.6 \%-8.6 \%$ [17]. In our study, although all the patients had different vascular ligation locations, they all underwent lymph node dissection of the inferior mesenteric artery. Postoperative pathology showed the total number of lymph node dissections, the number of positive lymph nodes and the total number of lymph nodes surrounding the inferior mesenteric artery did not significantly differ between these two groups. In addition, there was no metastasis in the lymph nodes at the root of the inferior mesenteric artery (253 set of lymph nodes) in these 35 patients. Postoperative pathology showed that the bowel length after fixation was significantly longer in the proximal group than in the distal group, which may be due to the lengthy sigmoid colon of some patients in the proximal group.

Clinically, the most serious complications after colon cancer surgery are intestinal obstruction, abdominal infection, and anastomotic leakage. Currently, it is believed that the main cause of anastomotic leakage is anastomotic tension and poor blood flow [18]. Relevant literature has pointed out that if the left colic artery is preserved intraoperatively in rectal cancer or sigmoid colon cancer resection, it can effectively protect the postoperative blood flow and inhibit the occurrence of anastomotic leakage, thereby improving the safety of the treatment $[13,18,19]$. In our study, there was no statistical difference in postoperative recovery time between the two groups. In addition, although the incidence of complications was lower in the proximal group than in the distal group, there was no significant difference. After analyzing the causes, we believe that the distal group included a greater proportion of patients who underwent high ligation of the inferior mesenteric artery, which may affect the anastomotic tension and blood supply.

The clinical significance of this study is its determination of the cutoff points of the distal and proximal sigmoid colon and comparison of the intraoperative conditions, postoperative pathology, and recovery of the two groups to achieve more precise and standardized laparoscopic radical resection of sigmoid colon cancer. If the sigmoid colon cancer is at the distal group, high ligation of the inferior mesenteric artery or preservation of the left colic artery is preferred during the surgery, stapler anastomosis might be performed, and an anal decompression tube might be retained. If the sigmoid colon cancer is located at the proximal group, surgery tends to retain the superior rectal artery, and manual anastomosis might be preferred. Of course, regardless of whether sigmoid colon cancer is distal or proximal, complete mesorectal excision and D3 lymph node dissection is the key to radical surgery $[16,17]$. In addition, anastomotic tension and blood supply are the most important factors influencing the occurrence of anastomotic leakage. Ensuring appropriate tension and 
adequate blood supply is particularly critical in colon anastomosis [18-20].

This study is based on a retrospective study with a small sample size, which can lead to bias in the research results; nonetheless, it provides important information and objectives for our next large-scale prospective study.

\section{Conclusion}

The patients with sigmoid colon tumor can be divided into distal group $(16-30 \mathrm{~cm}$ from the anus) and proximal group (31-50cm from the anus) according to the distance between the tumor and the anal margin. The patients in the distal group using stapler for intestinal anastomosis and anal decompression tube were significantly more than those in the proximal group. For the ligation of the inferior mesenteric artery, high ligation was usually used or the left colic artery was usually preserved in the distal group, and the superior rectal artery was usually retained in the proximal group.

\section{List of Abbreviations}

BMI: body mass index

CEA: carcinoembryonic antigen

AJCC: American Joint Committee on Cancer

\section{Declarations}

\section{-Ethics approval and consent to participate}

All methods were performed in accordance with the Declaration of Helsinki. This study was approved by the ethics committee of Sir Run Run Shaw Hospital Zhejiang University College of Medicine. The requirement for informed consent was waived by the ethics committee of Sir Run Run Shaw Hospital due to the retrospective nature of the study.

\section{-Consent for publication}

Written consent was obtained from the patient for the patient's personal or clinical details along with any identifying images to be published in this study.

\section{-Availability of data and materials}

The datasets generated and/or analyzed during the current study are not publicly available due to 
protecting individual patient privacy but are available from the corresponding author on reasonable request.

\section{-Competing interests}

Not applicable

\section{-Funding}

Not applicable

\section{-Author's contributions}

All authors have read and approved the manuscript.

Conceptualization: LC, ZFS

Data curation: LC, EGC, MC, WZ

Formal analysis: LC, EGC, MC, WZ

Methodology: LC, EGC, MC, WZ

Resources: LC, EGC, DAJ

Software: EGC

Writing - original draft: LC, MC, WZ

Writing - review \& editing: LC, EGC, ZFS

\section{-Acknowledgments}

This work was supported by the Zhejiang University College of Medicine. Our special thanks are due to Prof. He Chao and Huang Xuefeng for their helpful discussion with preparing the manuscript.

\section{References}

[1] Siegel RL, Miller KD, Fuchs HE, Jemal A. Cancer Statistics, 2021. CA Cancer J Clin. 2021 Jan;71(1):7-33.

[2] Bray F, Ferlay J, Soerjomataram I, Siegel RL, Torre LA, Jemal A. Global cancer statistics 2018: GLOBOCAN estimates of incidence and mortality worldwide for 36 cancers in 185 countries. CA Cancer J Clin. 2018;68(6):394-424.

[3] Benson AB, Venook AP, Al-Hawary MM, et al. Version 2.2018, NCCN clinical practice guidelines in oncology. J Natl Compr Canc Netw. 2018;16(7):874-901.

[4] Lin G, Qiu H. Patient selection and operation standard of transanal endoscopic microsurgery. Zhonghua Wei Chang Wai Ke Za Zhi. 2015 May;18(5):427-9.

[5] Daher R, Chouillard E, Panis Y. New trends in colorectal surgery: single port and natural orifice techniques. World J Gastroenterol. 2014 Dec 28;20(48):18104-20.

[6] Pascual M, Salvans S, Pera M. Laparoscopic colorectal surgery: Current status and implementation of the latest technological innovations. World J Gastroenterol. 2016 Jan 14;22(2):704-17. 
[7] Cirocchi R, Trastulli S, Farinella E, Desiderio J, Vettoretto N, Parisi A, Boselli C, Noya G. High tie versus low tie of the inferior mesenteric artery in colorectal cancer: a RCT is needed. Surg Oncol. 2012 Sep;21(3):e111-23.

[8] Ke J, Cai J, Wen X, Wu X, He Z, Zou Y, Qiu J, He X, He X, Lian L, Wu X, Zhou Z, Lan P. Anatomic variations of inferior mesenteric artery and left colic artery evaluated by 3-dimensional CT angiography: Insights into rectal cancer surgery - A retrospective observational study. Int J Surg. 2017 May;41:106-111.

[9] Yada H, Sawai K, Taniguchi H, Hoshima M, Katoh M, Takahashi T. Analysis of vascular anatomy and lymph node metastases warrants radical segmental bowel resection for colon cancer. World J Surg. 1997 Jan;21(1):109-15.

[10] Murono K, Kawai K, Kazama S, Ishihara S, Yamaguchi H, Sunami E, Kitayama J, Watanabe T. Anatomy of the inferior mesenteric artery evaluated using 3-dimensional CT angiography. Dis Colon Rectum. 2015 Feb;58(2):214-9.

[11] Bonnet S, Berger A, Hentati N, Abid B, Chevallier JM, Wind P, Delmas V, Douard R. High tie versus low tie vascular ligation of the inferior mesenteric artery in colorectal cancer surgery: impact on the gain in colon length and implications on the feasibility of anastomoses. Dis Colon Rectum. 2012 May;55(5):515-21.

[12] Crocetti D, Cavallaro G, Tarallo MR, Chiappini A, Polistena A, Sapienza P, Fiori E, De Toma G. Preservation of left colic artery with lymph node dissection of IMA root during laparoscopic surgery for rectosigmoid cancer. Results of a retrospective analysis. Clin Ter. 2019 Mar-Apr;170(2):e124-e128.

[13] Si MB, Yan PJ, Du ZY, Li LY, Tian HW, Jiang WJ, Jing WT, Yang J, Han CW, Shi XE, Yang $\mathrm{KH}$, Guo TK. Lymph node yield, survival benefit, and safety of high and low ligation of the inferior mesenteric artery in colorectal cancer surgery: a systematic review and meta-analysis. Int J Colorectal Dis. 2019 Jun;34(6):947-962.

[14] Eiholm S, Ovesen H. Total mesocolic excision versus traditional resection in right-sided colon cancer - method and increased lymph node harvest. Dan Med Bull. 2010 Dec;57(12):A4224.

[15] Palumbo P, Usai S, Pansa A, Lucchese S, Caronna R, Bona S. Anastomotic Leakage in Rectal Surgery: Role of the Ghost Ileostomy. Anticancer Res. 2019 Jun;39(6):2975-2983.

[16] Wong JH, Severino R, Honnebier MB, Tom P, Namiki TS. Number of nodes examined and staging accuracy in colorectal carcinoma. J Clin Oncol. 1999 Sep;17(9):2896-900.

[17] Chin CC, Yeh CY, Tang R, Changchien CR, Huang WS, Wang JY. The oncologic benefit of high ligation of the inferior mesenteric artery in the surgical treatment of rectal or sigmoid colon cancer. Int J Colorectal Dis. 2008 Aug;23(8):783-8.

[18] Komen N, Slieker J, de Kort P, de Wilt JH, van der Harst E, Coene PP, Gosselink MP, Tetteroo G, de Graaf E, van Beek T, den Toom R, van Bockel W, Verhoef C, Lange JF. High tie versus low tie in rectal surgery: comparison of anastomotic perfusion. Int J Colorectal Dis. 2011 Aug;26(8):1075-8.

[19] Zeng J, Su G. High ligation of the inferior mesenteric artery during sigmoid colon and rectal cancer surgery increases the risk of anastomotic leakage: a meta-analysis. World J Surg Oncol. 2018 Aug 2;16(1):157.

[20] Kanemitsu Y, Hirai T, Komori K, Kato T. Survival benefit of high ligation of the inferior mesenteric artery in sigmoid colon or rectal cancer surgery. Br J Surg. 2006 May;93(5):609-15. 


\section{Figure Legend}

\section{Figure}

\section{Classification of inferior mesenteric artery}

In type $\mathrm{A}$, the inferior mesenteric artery first divides into the left colonic artery, and the sigmoid artery and the superior rectal artery share the same trunk; in type B, the left colonic artery and the sigmoid artery share the same trunk; in type $\mathrm{C}$, the inferior mesenteric artery divides into the left colonic artery, the sigmoid artery and the superior rectal artery at the same point

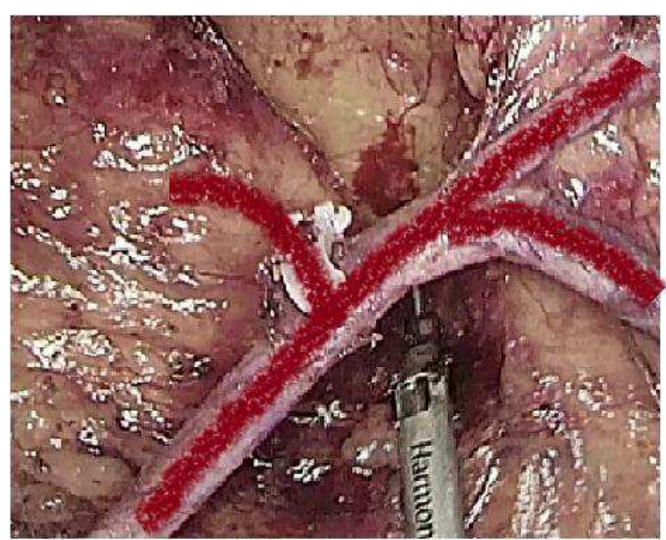

A

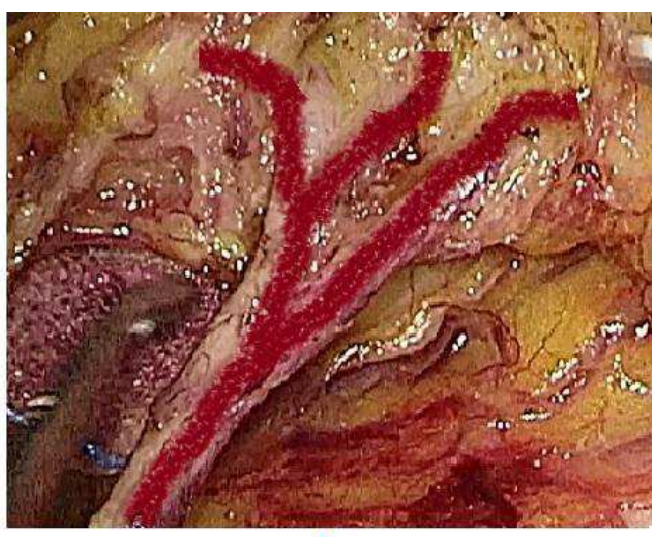

B

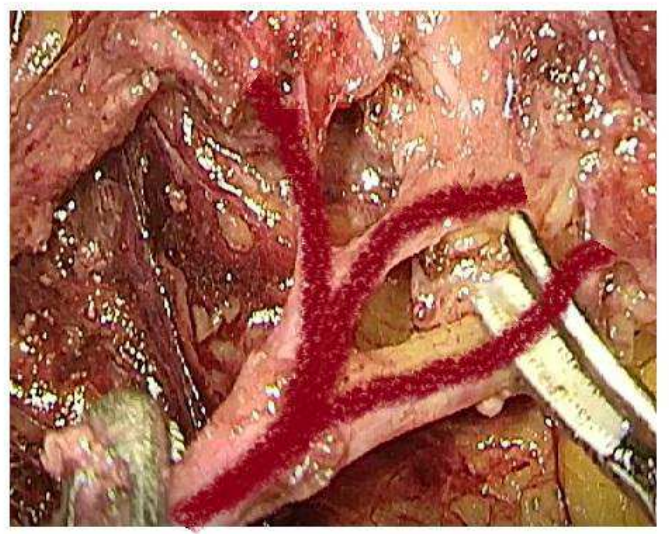

C

\section{Figure}

Classification of inferior mesenteric artery

In type $A$, the inferior mesenteric artery first divides into the left colonic artery, and the sigmoid artery and the superior rectal artery share the same trunk; in type $B$, the left colonic artery and the sigmoid artery share the same trunk; in type $\mathrm{C}$, the inferior mesenteric artery divides into the left colonic artery, the sigmoid artery and the superior rectal artery at the same point 
Table 1 Selection of the cutoff points

for the location of sigmoid colon cancer

\begin{tabular}{cccc}
\hline $\begin{array}{c}\text { Location of sigmoid } \\
\text { colon cancer }(\mathbf{c m})\end{array}$ & $\begin{array}{c}\text { Number of the } \\
\text { patients }\end{array}$ & $\begin{array}{c}\text { Distance between the tumor and } \\
\text { the anal margin }(\mathbf{c m}) \overline{\mathbf{x}} \pm \mathbf{s}\end{array}$ & $\mathbf{p}$ \\
\hline $16-20$ & 11 & $15.27 \pm 0.86$ & $7.44 * 10^{-5}$ \\
$21-50$ & 24 & $28.75 \pm 9.58$ & \\
\hline $16-25$ & 19 & $17.26 \pm 2.42$ & $3.02 * 10^{-8}$ \\
$26-50$ & 16 & $33.13 \pm 8.95$ & $6.76 * 10^{-11}$ \\
\hline $16-30$ & 24 & $18.67 \pm 3.53$ & \\
$31-50$ & 11 & $37.27 \pm 7.79$ & $1.48 * 10^{-10}$ \\
\hline $16-35$ & 29 & $20.62 \pm 5.35$ & \\
\hline $36-50$ & 6 & $43.33 \pm 5.53$ & \\
\hline
\end{tabular}

Table 2 Clinical characteristics of this study

\begin{tabular}{lccc}
\hline \multicolumn{1}{c}{ Variable } & $\begin{array}{c}\text { Distal group } \\
\mathbf{n = 2 4}\end{array}$ & $\begin{array}{c}\text { Proximal group } \\
\mathbf{n = 1 1}\end{array}$ & $\mathbf{p}$ \\
\hline Age $\quad \overline{\mathrm{x}} \pm \mathrm{s}$ & $62.42 \pm 10.16$ & $65.27 \pm 11.23$ & 0.474 \\
Male $(\mathrm{No}, \%)$ & $14,58.3 \%$ & $9,81.8 \%$ & 0.330 \\
$\mathrm{BMI}\left(\mathrm{kg} / \mathrm{m}^{2}\right) \overline{\mathrm{x}} \pm \mathrm{s}$ & $24.40 \pm 3.93$ & $23.41 \pm 2.22$ & 0.453 \\
$\mathrm{CEA}(\mathrm{ng} / \mathrm{ml}) \overline{\mathrm{x}} \pm \mathrm{s}$ & $5.12 \pm 4.16$ & $10.89 \pm 8.92$ & $0.019^{*}$ \\
Distance between the tumor and & $18.67 \pm 3.53$ & $37.27 \pm 7.79$ & $<0.001^{*}$ \\
the anal margin $(\mathrm{cm}) \overline{\mathrm{x}} \pm \mathrm{s}$ & & & \\
\hline
\end{tabular}


Table 3 Intraoperative situation

\begin{tabular}{lccc}
\hline \multicolumn{1}{c}{ Variable } & $\begin{array}{c}\text { Distal group } \\
\mathbf{n = 2 4}\end{array}$ & $\begin{array}{c}\text { Proximal group } \\
\mathbf{n = 1 1}\end{array}$ & $\mathbf{p}$ \\
\hline Using stapler anastomosis (No, \%) & $21,87.5 \%$ & $3,12.5 \%$ & $0.002^{*}$ \\
Artery ligation (No, \%) & & & $<0.001^{*}$ \\
$\quad$ High ligation & $10,41.7 \%$ & $2,18.2 \%$ & \\
$\quad$ Retaining left colic artery & $11,45.8 \%$ & 0 & \\
$\quad$ Retaining superior rectal artery & $3,12.5 \%$ & $9,81.8 \%$ & \\
Vascular management time (min) $\overline{\mathrm{x}} \pm \mathrm{s}$ & $24.38 \pm 9.17$ & $27.36 \pm 13.20$ & 0.458 \\
Surgical duration (min) $\overline{\mathrm{x}} \pm \mathrm{s}$ & $183.54 \pm 59.68$ & $200.91 \pm 92.22$ & 0.522 \\
Intraoperative blood loss (ml) $\overline{\mathrm{x}} \pm \mathrm{s}$ & $100.00 \pm 193.74$ & $110.91 \pm 187.10$ & 0.880 \\
Using anal decompression tube $(\mathrm{No}, \%)$ & $20,83.3 \%$ & $3,12.5 \%$ & $0.004^{*}$ \\
\hline
\end{tabular}

Table 4 Pathological analysis

\begin{tabular}{lccc}
\hline \multicolumn{1}{c}{ Variable } & $\begin{array}{c}\text { Distal group } \\
\mathbf{n = 2 4}\end{array}$ & $\begin{array}{c}\text { Proximal group } \\
\mathbf{n = 1 1}\end{array}$ & p \\
\hline Tumor pathological stage $(\mathrm{No}, \%)$ & & & 0.063 \\
0 & $2,8.3 \%$ & $2,18.2 \%$ & \\
I & $3,12.5 \%$ & 0 & \\
II & $5,20.9 \%$ & $5,45.4 \%$ & \\
III & $12,50 \%$ & $1,9.1 \%$ & \\
IV & $2,8.3 \%$ & $3,27.3 \%$ & \\
Pathological differentiation (No, \%) & & & 0.941 \\
Low differentiated & $9,37.5 \%$ & $5,45.5 \%$ & \\
Moderate and high differentiated & $15,62.5 \%$ & $6,54.5 \%$ & \\
Length of the bowel specimen (cm) $\overline{\mathrm{x}} \pm \mathrm{s}$ & $10.72 \pm 2.57$ & $13.43 \pm 2.29$ & $0.006 *$ \\
Total number of lymph node dissections $\overline{\mathrm{x}} \pm \mathrm{s}$ & $17.88 \pm 5.91$ & $16.55 \pm 4.52$ & 0.525 \\
Total number of positive lymph nodes $\overline{\mathrm{x}} \pm \mathrm{s}$ & $1.29 \pm 1.72$ & $0.36 \pm 0.77$ & 0.106 \\
Number of dissections at the root of the & $2.75 \pm 1.85$ & $3.75 \pm 4.21$ & 0.614 \\
inferior mesenteric vessels $\overline{\mathrm{x}} \pm \mathrm{s}$ & & & \\
\hline
\end{tabular}


Table 5 Postoperative recovery and complications

\begin{tabular}{lccc}
\hline \multicolumn{1}{c}{ Variable } & $\begin{array}{c}\text { Distal group } \\
\mathbf{n = 2 4}\end{array}$ & $\begin{array}{c}\text { Proximal group } \\
\mathbf{n = 1 1}\end{array}$ & $\mathbf{p}$ \\
& $2.48 \pm 0.71$ & $2.55 \pm 0.50$ & 0.787 \\
\hline Time to exhaust $(\mathrm{d}) \overline{\mathrm{x}} \pm \mathrm{s}$ & $4.26 \pm 0.99$ & $4.27 \pm 0.75$ & 0.973 \\
Time to fluid intake (d) $\overline{\mathrm{x}} \pm \mathrm{s}$ & $8.17 \pm 3.73$ & $8.73 \pm 2.09$ & 0.659 \\
Drainage tube removal time (d) $\overline{\mathrm{x}} \pm \mathrm{s}$ & $10.71 \pm 4.74$ & $10.27 \pm 2.63$ & 0.783 \\
Postoperative hospital stay (d) $\overline{\mathrm{x}} \pm \mathrm{s}$ & $4,16.7 \%$ & $1,9.1 \%$ & 0.941 \\
Complications (No, \%) & & & \\
\hline
\end{tabular}

